\title{
Ventriculectomia Parcial em Criança - Técnica de Batista
}

\author{
Luiza Mara Elias, Raynold Monteiro, Rosângela Fittaroni, Marcelo Zaninotto, Eduardo Coelho de \\ Souza, Maria Alejandra Rivero, Daniela Chaves, Randas José Vilela Batista, Magnus Coelho de Souza
}

São Paulo, SP - Campina Grande do Sul, PR

\begin{abstract}
Criança com dois anos e seis meses de idade, apresentando cardiomiopatia dilatada idiopática, classe funcional III-IV (New York Heart Association - NYHA) e indicação de transplante cardíaco, foi submetida à ventriculectomia parcial, pela técnica de Batista. A avaliação pré-operatória com ecocardiograma $(E C O) e$ ventriculografia radioisotópica (Gated Blood Pool) revelaram fração de ejeção de ventrículo esquerdo (FEVE) de $13 \%$ e 20\%, respectivamente. Após a cirurgia, que consistiu na remoção de uma parte do músculo da parede lateral do ventrículo esquerdo, a FEVE foi para $50 \%$ pelo ECO e $30 \%$ pela ventriculografia radioisotópica. A criança recebeu alta hospitalar no $16^{\circ}$ pós-operatório em classe funcional I (NYHA).
\end{abstract}

\section{Partial Ventriculectomy in a Child - the Batista Operation}

We present the case of a two and a half year old child with idiopatic dilated cardiomyopathy and end-stage congestive heart failure (NYHA III-IV) who underwent "partial ventriculectomy". The operation was performed under normothermic cardiopulmorary bypass and normothermic oxygenated blood cardioplegia and consisted of removal of a large wedge of muscle from the lateral wall of the left ventricle beginning at the apex and extending between the papillary muscles, ending proximal to the mitral annulus. Pre-operative the left ventricular ejection fraction (EF) was $13 \%$ by echocardiography and $20 \%$ by radionuclide ventriculography. Postoperative avaliation showed improvement of EF to $50 \%$ and $30 \%$, respectively. The child was in functional class I (NYHA) on discharge from hospital (16 $6^{\text {th }}$ postoperative day).

Arq Bras Cardiol, volume 69 (n 2), 133-135, 1997

O tratamento cirúrgico de pacientes com miocardiopatia em fase terminal tinha como opções terapêuticas a cardiomioplastia, o transplante cardíaco, os dispositivos de assistência ventricular e o coração artificial. A partir de 1984, com a técnica proposta por Batista, a ventriculectomia parcial, que consiste na retirada da musculatura da parede lateral do ventrículo esquerdo (VE), entre os músculos papilares ${ }^{1}$, o prognóstico de pacientes com miocardiopatia terminal e em protocolo de transplante cardíaco mudou, com melhora clínica e hemodinâmica significantes. Devido aos bons resultados multicêntricos obtidos, e à simplicidade e fácil reprodução da técnica, optamos por realizá-la em uma criança de dois anos e seis meses de idade com cardiomiopatia dilatada idiopática, classe funcional III-IV (NYHA) e

Hospital Beneficência Portuguesa - São Paulo e Centro Médico Caron - Campina Grande do Sul

Correspondência: Luiza Mara Elias - Hospital Beneficência Portuguesa - Rua Maestro Cardim, 769 - $2^{\circ}$ - S/264 - 01323-001 - São Paulo, SP

Recebido para publicação em 23/6/97

Aceito em 29/7/97 indicação de transplante cardíaco. O resultado excelente e a ausência de descrição literária desta técnica em crianças, estimulou-nos a relatar o presente caso.

\section{Relato do Caso}

Paciente do sexo masculino, com dois anos e seis meses de idade, $12 \mathrm{~kg}, 86 \mathrm{~cm}$ de altura, parda, vacinação completa, cardiopatia diagnosticada aos três meses de idade. Mãe relata dispnéia aos mínimos esforços e em repouso, acompanhada de palidez, sudorese e tontura. Apresentou infecção de vias aéreas superiores de repetição e um episódio de pneumonia, na época da $1^{\mathrm{a}}$ consulta. Ao exame físico apresentava-se descorado (+/4+), dispnéico, frequiência cardíaca com ritmo regular de $98 \mathrm{bpm}$ com sopro sistólico em foco mitral irradiado para axila $(+/ 4+)$, pressão arterial 95/ $65 \mathrm{mmHg}$, ausculta pulmonar com roncos bilaterais e estertores inspiratórios em base esquerda, abdômen flácido, indolor, com fígado palpável a $5 \mathrm{~cm}$ do rebordo costal direito. Radiografia de tórax mostrando aumento global de área cardíaca $(++/ 4+)$; eletrocardiograma com sobrecarga biven- 


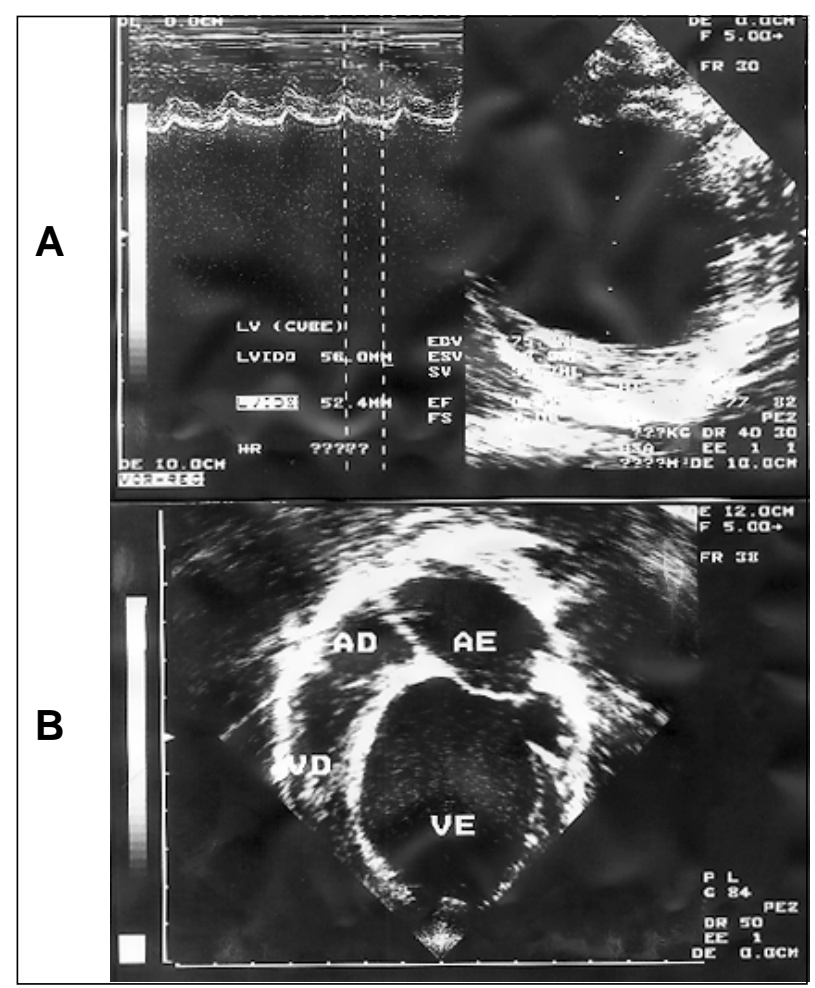

Fig. 1 - A) Ecocardiografia modo-M de pré-operatório, mostrando fração de ejeção de 13\%; B) ecocardiograma bidimensional apical 4 câmaras, de pré-operatório, mostrando aumento acentuado de VE.

tricular, predominando em VE; ecocardiograma (ECO) com diagnóstico de cardiomiopatia dilatada importante, com insuficiência mitral moderada, fração de ejeção de ventrículo esquerdo (FEVE) 13\%, diâmetro diastólico do ventrículo esquerdo(DDVE) 61mm, diâmetro sistólicodo ventrículoesquerdo(DSVE) $53 \mathrm{~mm}$, aorta (Ao) $18 \mathrm{~mm}$, átrioesquerdo(AE) $28 \mathrm{~mm}$, delta D 7\% (fig. 1A e 1B). À ventriculografia radioisotópica, a FEVE era de $20 \%$ (normal > 46\%) e a fração de ejeção de ventrículodireito (FEVD) de $41 \%$ (normal $>40 \%$ ). Aguardoua cirurgia em uso de digital, diurético e captopril.

Em 20/5/97 realizamos a ventriculectomia parcial pela técnica de Batista, através de uma esternotomia mediana e circulação extracorpórea (CEC) em normotermia, após canulação bicaval e aórtica. A Ao foi pinçada e, em seguida, injetada solução cardioplégica sangüínea normotérmica (Miranda) nas coronárias. A cirurgia consistiu na remoção de duas fatias de músculo da parede lateral do VE, iniciandose na ponta do mesmo, estendendo-se entre os músculos papilares e terminando próximo ao anel mitral (fig. 2A e 2B). Os retalhos musculares foram enviados para estudo anatomopatológico, que revelou fibrose miocárdica com hipertrofia de fibras musculares e fibrose intersticial. A ventriculectomia foi fechada com sutura contínua de polipropilene 000 em dois planos (fig. 3). Após retirada cuidadosa do ar intracavitário e abertura da pinça da Ao, o coração recuperou os batimentos com arritmias ventriculares. Saiu de CEC com drogas antiarrítmicas e marcapasso em demanda. Não houve necessidade do uso de anti-hipertensivos. A criança foi encaminhada à UTI, acordada, podendo em seguida ser extubada. Realizado ECO no pós-operatório (PO) imediato, que mostrou uma FEVE de 39\%; DDVE 42mm;
DSVE37mm; Ao 17mm;AE25mm; deltaD18\%.Evoluiu com agitação e febre que não cedia aos antitérmicos. Foram colhidas três amostras de sangue para hemocultura, as quais apresentaram-se negativas, juntamente com hemogramas e radiografia de tórax normais. No $3^{\circ}$ dia $\mathrm{PO}$ apresentou crise convulsiva. Realizada tomografia computadorizada de crânio, foi diagnosticada hemorragia meníngea na fissura interhemisférica posterior e sobre o tentório. A criança mantinha-se febril, mas sem seqüelas neurológicas. Foram feitas novas hemoculturas e exame de líquor, todos negativos. Optamos pela não utilização de antibióticos, por julgar ser a febre de origem central. No $5^{\circ}$ dia PO, evoluiu com hemograma infeccioso e, à radiografia, imagem de condensação em base de pulmão esquerdo. Iniciada antibioticoterapia com ceftriaxona, mantida por 10 dias. Recebeu alta da UTIno $7^{\circ}$ dia PO e alta hospitalar no $16^{\circ}$. Realizado ECO tardio $\left(15^{\circ}\right.$ PO) que revelou FEVE 50\% (fig. 4Ae 4B) e ventriculografia radioisotópica que mostrou FEVE $30 \%$. A criança deixou o hospital em classe funcional I (NYHA) e sem seqüelas neurológicas, com digital, diurético e captopril .

\section{Discussão}

A cardiomiopatia dilatada idiopática é a mais comum, dentre as outras formas de cardiomiopatia. Em crianças, expressa-se pela insuficiência cardíaca congestiva e baixo débito cardíaco, tendo como principais complicações as arritmias cardíacas e embolias sistêmicas e pulmonares ${ }^{2}$. Devido ao elevado índice de mortalidade, o transplante cardíaco tornou-se a principal opção terapêutica ${ }^{2}$.

O transplante cardíaco infantil foi realizado, pela pri-

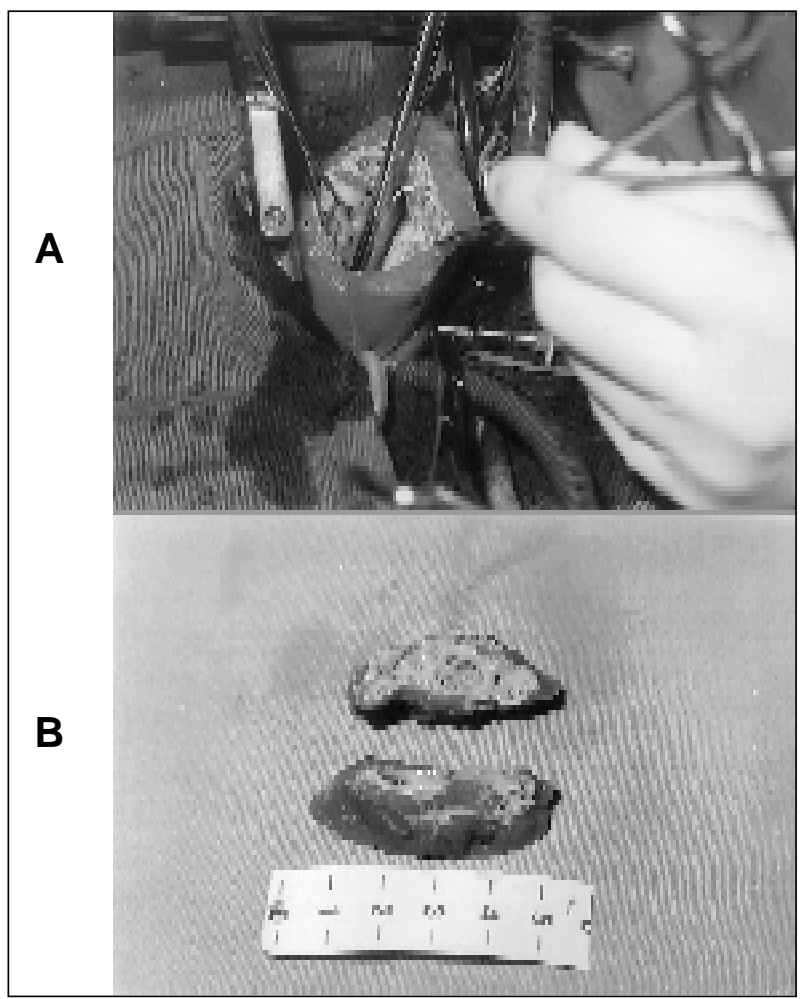

Fig. 2 - A) Ventrículo esquerdo após retirada de musculatura da parede lateral; B) fatias musculares retiradas do VE, com fibrose miocárdica. 


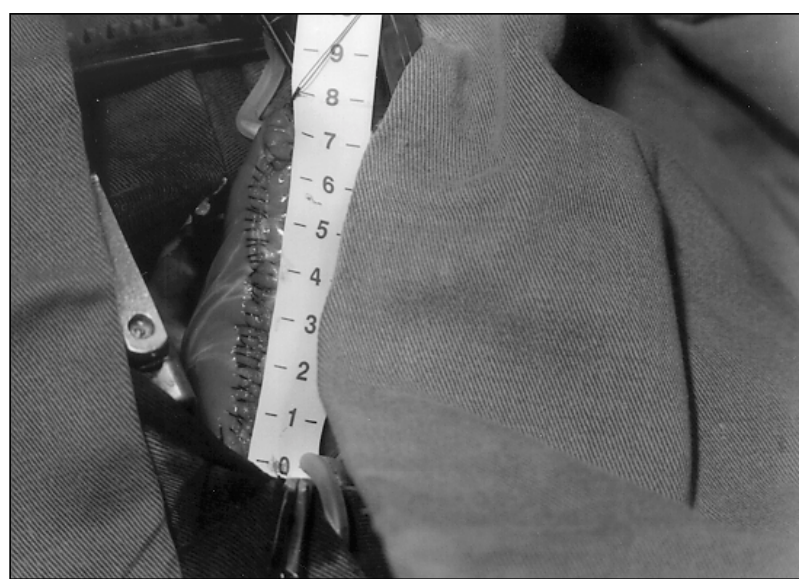

Fig. 3 - Sutura do ventrículo esquerdo com fio polipropilene 000, em 2 planos.

meira vez, poucas semanas após o transplante cardíaco em adulto, por Kantrowitz e col ${ }^{3}$, em uma criança de 16 dias, com anomalia de Ebstein. Em nosso meio, os resultados são favoráveis ( $85,7 \%$ de sobrevida a médio prazo), mas a mortalidade é grande enquanto se aguarda na fila de espera $(60 \%)^{4}$.

A cardiomioplastia foi realizada pela primeira vez por Carpentier, em 1985, em um paciente com miocardiopatia, classe funcional IV (NYHA) e em programa de transplante cardíaco ${ }^{5}$. Esta melhora a condição clínica e a função ventricular esquerda de pacientes com cardiomiopatia dilatada, mas os resultados são limitados pela elevada mortalidade tardia, em pacientes operados em classe funcional IV (NYHA). Também tem como complicações a isquemia do enxerto muscular no PO imediato e a possibilidade de degeneração gordurosa desse enxerto a longo prazo, deixando dúvidas quanto à validade terapêutica deste procedimento ${ }^{6}$. Não encontramos relatos na literatura médica de cardiomioplastia em crianças.

Os dispositivos de assistência ventricular estão disponíveis, desde 1963, aos pacientes candidatos a transplante cardíaco (ponte para transplantes) e aos pacientes com quadro agudo de falência miocárdica, com objetivo de manter as condições cardiocirculatórias estáveis. Em adultos, já se encontram dificuldades, principalmente pela falta de padronização e controle hemodinâmico dos pacientes ${ }^{7}$.

A ventriculectomia parcial, desenvolvida a partir de 1984, por Batista, soma-se aos recursos terapêuticos disponíveis pelos cirurgiões cardiovasculares no tratamento da cardiomiopatia e miocardiopatia terminais.

Devido à melhora na qualidade de vida proporcionada aos pacientes adultos, optamos pelo uso desta técnica também em crianças, iniciando em uma de dois anos e seis
Ventriculectomia parcial em criança

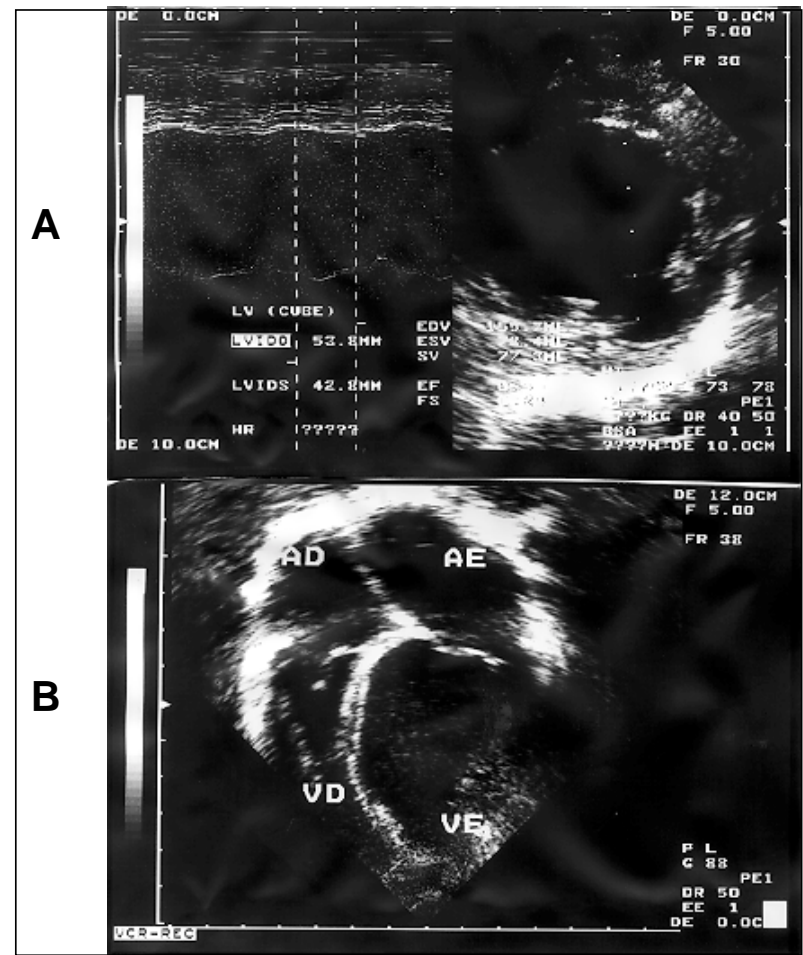

Fig. 4 - A) Ecocardiografia modo-M de pós-operatório tardio (15²), mostrando fração de ejeção de $50 \%$; B) ecocardiograma bidimensional apical 4 câmaras de pós-operatório tardio $\left(15^{\circ}\right)$, mostrando redução da cavidade ventricular esquerda.

meses de idade, com cardiomiopatia dilatada idiopática.

A melhora clínica e hemodinâmica foram bem evidentes na avaliação pós-operatória (exame clínico, ECO e Gatted).

Observamos também que o paciente, adulto ou criança, tem o "momento cirúrgico" de indicação da técnica, o que está na dependência de variáveis, para obtenção do melhor resultado cirúrgico, como: classe funcional, comprometimento de outros órgãos e grau de hipertensão pulmonar.

Pesquisando na literatura médica indexada, não encontramos nenhum trabalho referente ao tratamento cirúrgico da cardiomiopatia dilatada em crianças, através da técnica de Batista.

Em conclusão, podemos dizer que esta técnica é simples e facilmente reprodutível, também em crianças. Os resultados iniciais são animadores, tornando a expectativa muito grande, com relação aos resultados a longo prazo, mas acreditamos que, somente pela grande melhora clínica que os pacientes obtêm, e em último caso, podendo prolongar o tempo de espera em uma fila de transplantes, faz com que esta técnica seja uma das grandes contribuições à cirurgia cardíaca nos últimos anos.

\section{Referências}

1. Batista RJV, Santos JLV, Franzoni M et al - Ventriculectomia parcial: um novo conceito no tratamento cirúrgico de cardiopatias em fase final. Rev Bras Cir Cardiovasc 1966; 11: 1-6.

2. Camargo P, Snitcowsky R, Mazzieri R, Ebaid M-Cardiopatias infantis. Rev Soc Cardiol ESP 1992; 1: 52-8.

3. Kantrowitz A, Haller JD, Joos H et al - Transplantation of the heart in a infant and an adult. Am J Cardiol 1968; 22: 782-90.

4. Barbero-Marcial M, Azeka E, Camargo PR et al-Características do transplante cardíaco neonatal e infantil. Rev Bras Cir Cardiovasc 1996; 11 : 60-6.

5. Carpentier A - Myocardial substitution with stimuleted skeletal muscle: first successful clinical case. Lancet 1985; 1: 1267.

6. Moreira LFP, Stolf NAG, Bocchi EA et al - Evolução clínica e comportamento da função ventricular no pós-operatório tardio da cardiomioplastia. Rev Bras Cir Cardiovasc 1995; 10: 3-17.

7. Sezae Y - Artificial Heart. Tokio: Harcourt Brace Jovanovich Japan, 1992: 23 